\title{
Node Density Based Performance Comparison of Neighbor Knowledge and Probability Based Broadcast Approaches With Conventional Routing Protocols In MANET's
}

\author{
E.Gnanamanoharan, \\ Department of ECE, \\ Annamalai University, \\ gnanamanohar@gmail.com
}

\begin{abstract}
Wireless Mobile Ad hoc networks have a number of advantages over their traditional wired counterparts. They can be deployed in areas without a pre-existing wired. Due to the random mobility of nodes, the topology of the network changes often. This is an important aspect in the design and effective routing algorithm which should be efficient and consistent to discover new routes and maintain them till the successful delivery of packets between the node pairs. A highly topology adaptable ad hoc routing protocol that used to detect and repair the link breakage in dynamic topology. MANET routing protocols are challenged with establishing and maintaining multi-hop routes due to frequent mobility speed. This is an important aspect in the design of effective routing algorithm which should be efficient and consistent to discover new routes and maintain them till the successful delivery of packets between the node pairs. This paper provides node density based performance comparison of neighbor knowledge and probability based broadcast approaches with proactive and reactive routing protocols. The simulations are carried out to pinpoint various performance issues and comparison between them.
\end{abstract}

Keywords - MANETs, routing protocol, reactive, proactive, hybrid, broadcasting techniques, dynamic topology.

\section{INTRODUCTION}

MANET Routing protocol is a set of rules which monitors the traversing of message packets from source to destination in a network. MANET has different types of routing protocols and each of them is applied according to the network circumstances. The major types of routing protocols are proactive, reactive and hybrid. Proactive (or Table driven) routing protocols attempt to maintain up-todate routing information to all nodes by periodically disseminating topological updates throughout the network and thus suffer the disadvantage of additional control traffic that is needed to continually update stale route entries. Reactive (or On-demand) routing protocols attempt to discover a route only when a route is needed and Hybrid protocols inherit the features from both proactive and reactive routing protocols. The topology of the network changing often or quickly due to mobility of the nodes and is an important aspect in the design of effective routing algorithm which should be efficient and consistent to discover new routes and maintain them till the successful delivery of packets between appropriate source destination pair. The mobility of a node is always been one of the important characteristics in determining the overall performance of the Ad hoc network.

\section{REVIEW OF THE LITERATURE}

Perkins and Bhagwat, (1994) developed DSDV reactive routing protocol to calculate the shortest number of hops to the destination. There is increasing of routing overhead due to exchange of routing information in dynamic network scenarios and solutions are given for issues related to route discovery and maintenance. Perkins and Royer, (1999) developed the Ad hoc On-demand Distance Vector (AODV) and this is one of the most significant contributions to MANET routing.
I.

Jose Moses et al (2012) evaluated the performance of AODV, DSR and DSDV with Constant Bit Rate (CBR) traffic and it is effective for scalable performance with 40 nodes. Mehmood, (2014) has given a comprehensive performance analysis of DSR, AODV, and DSDV routing protocol for different metrics in different scenarios.

Mohammed et al (2009) conducted comprehensive simulation study on the multipath routing protocols for mobile Ad hoc networks. Deepak and Yogesh, (2011) presented a probabilistic broadcasting algorithm based on traffic analysis. Manickam et al (2011) analyzed the performance of the three well known protocols AODV, DSR and DSDV with respect to variable node density.

Camp et al (2002) discussed the salient feature mobility models to established a relationship between path duration and MANET design parameters including node density, transmission range, number of hops and velocity of nodes. Divecha et al (2007) analyzed the performance of DSR and DSDV routing protocols with different mobility models. Ni et al (1999) discussed the issues of broadcast storm problem due to blind flooding which leads to degradation of entire network performance.

Cartigny and Simplot, (2003) proposed an algorithm which combines the advantages of both probabilistic and distance methods to privilege the retransmission by nodes that are located at the radio border of the sender. Zhang and Agrawal, (2004) proposed a scheme that reduces blind flooding by fixing the probability high when receiving a broadcast packet for the first time in the network. Kim et al (2004) described a probabilistic method for on demand route discovery, where the probability to forward an RREQ packet is determined by the number of duplicate RREQ packets received at a node.

Abdulai et al (2007) investigated the effects of pause time setting for AODV routing protocol using Random Point Group mobility model (RPGM). Abdalla et al (2008) 
proposed a dynamic probabilistic broadcasting scheme for MANETs where nodes move according to way point mobility model and this approach dynamically sets the value of the rebroadcast probability for every host node according to the neighborhood information.

\section{BROADCASTING METHODS}

Broadcasting is an essential building block of any MANET, so it is imperative to utilize the most efficient broadcast methods possible to ensure a reliable network. Broadcasting MANET [9] poses more challenges than in wired networks due to node mobility and scarce system resources. Because of the mobility there is no single optimal scheme for all scenario. Broadcasting methods have been categorized into four families utilising the IEEE 802.11 MAC specifications. Simple flooding requires each node in a MANET to rebroadcast all packets and probability based [4] assigns probabilities to each node to rebroadcast depending on the topology of the network. Area based common transmission distance is assumed and a node will rebroadcast if there is sufficient coverage area and Neighborhood based[19], State on the neighborhood is maintained by neighborhood method, the information obtained from the neighboring nodes is used for rebroadcast Apart from simple flooding, each broadcasting category aims at optimizing energy and bandwidth by minimizing message retransmission.

\subsection{Proactive and Reacive routing protocols (AODV and DSDV)}

AODV is a reactive or source initiated on-demand protocol which requires that all mobile nodes obtain routes as needed with little or no reliance on periodic advertisements[8]. It has been described as a pure ondemand route acquisition system because when connectivity is required, each host becomes aware of its neighbours by the use of hello messages and a path discovery process is initiated to locate the destination host. It is based on distance vector and does not require any nodes to maintain routes to destination and composed into two important phases are "route discovery" [10] and "route maintenance", which work together to permit discover and maintain routes for appropriate pair of source and destination and need an optimum path for the reliable delivery of data packets.

DSDV is a best known protocol for a proactive routing scheme based on distance vector and routing decision taken by hop count as cost metric [21]. The basic improvements are made to include freedom from loops in routing tables for more dynamic and less convergence time. It requires each node needed to be periodically broadcasting the routing updates and utilize a sequence number to tag every route. Each node maintains a routing table which contains list of all known destination nodes within the network along with number of hops required to reach to particular node. Each entry is marked with a sequence number assigned by the destination node. It requires adequate time to converge before the route can be used and protocol exhibits a shorter delay because it's a kind of table-driven routing protocol [20]. Each node maintains a routing table in which all of the possible destinations are within the network and the number of hops to each destination is recorded. Only packets belonging to valid routes at the ending instant get through.
A lot of packets are lost until new (valid) route table entries have been propagated through the network by the route update messages in DSDV.

\subsection{Broadcast Neighbor Discovery Scheme (BNDS)}

Broadcast Neighbor Discovery Scheme (BNDS) is a new broadcasting approach to minimize the effect of flooding by reducing the redundant broadcast and thus the routing overhead is also reduced. In BNDS [17], each node maintains a parameter as neighbor degree which is the largest number of one hop neighbors with source node. The source node selects a subset of its neighbors for forwarding the packet being broadcasted to additional nodes based on the neighbor degree. The aim of this scheme is to minimize the effect of flooding by reducing redundant broadcasts and reduces the routing overhead. Each node keeps knowledge of their neighbors within one hop and two hops radius that is accomplished by periodic hello messages. Each message contains the node address and a list of known neighbors. When a client receives a "hello" packet [15] from all its neighbors, it accepts a two-hop topology information i.e. only packets that would reach additional neighbors are rebroadcast [16] and select a particular set of the node to forward the broadcast packet to their one-hop neighbors. The sender chooses group of one hop neighbors for forwarding the broadcast packet includes their addresses in the packet header and broadcasts the packet. Those particular set of nodes that receive the broadcast packets are forwarding nodes if the address is contained in the packet header. Otherwise, it simply drops the received packet. Neighbor nodes repeat the same process carried by the sender. The selection of forwarding node based on the largest number of neighbors among one hop neighbors with the sender is called neighbor degree, if an intermediate node. When an RREQ packet reaches its destination node, the destination sends a reply to the source of the request, and it does not forward the packet. Information on neighbors that used in the proposed schemes is obtained via "hello" messages that are exchanged periodically similar with conventional AODV.

Each node follows a parameter called the node degree $d$, where the degree of node $\mathrm{N}$, degree $(\mathrm{N})$, is the number of one-hop neighbors with this node. The degree of a node is equal to the size of that nodes neighbor table and this table contains an entry for each neighbor updated from within specified time intervals. The merit of the degree considered as the largest number one hop neighbor in the network. Each node broadcasts a "hello" message containing its address and degree and a node frequently updates its routing and neighbor tables containing the addresses of all nodes one-hop neighbors and their degrees sorted in the decreasing order. If a source node $S$ wants to transmit the data packet to destination $\mathrm{D}$, it starts sending RREQ to its nearest one hop neighbors and need to choose first three or four forwarding nodes based on the order of largest degree. There is further need to optimize the act of forwarding nodes $(F)$ depending on the density of the nodes. $F$ is chosen as three in this work. The source node appends their addresses in the RREQ message. Upon receiving the RREQ message, only those nodes whose addresses are among the address of $\mathrm{F}$ neighbors will process the message and rebroadcast it further. 


\subsection{Probabilistic Broadcast Ad Hoc On-Demand Distance Vector (PBAODV)}

On-demand routing protocols discover a route between source destination pair with help neighbors information and they never need of topological information about the entire network, and thus there is no periodic update of routing information but efficient route discovery approach is very important to improve the network performance. When a sender needs a route to some destination, it broadcasts a RREQ packet to its one hop neighbors. Every neighboring node rebroadcasts the received RREQ packet [3] only once if it has no valid route to the destination. Each intermediate node that forwards the RREQ packet creates a reverse route pointing towards the sender. When the desired destination node or an intermediate node with a valid route to the destination receives the RREQ packet, it replies by sending a route reply (RREP) packet. The RREP packet is unicast towards the sender along the reverse path set-up by the forwarded RREQ packet. In traditional AODV, an intermediate node rebroadcasts all RREQ packets that are received for the first time. Assuming no intermediate node has a valid route to the destination and $\mathrm{N}_{\mathrm{t}}$ is the total number of nodes in the network, the number of possible rebroadcast in AODV is $N_{t}-1$. The basic probabilistic broadcast route discovery is simple. A source node sends an RREQ to its immediate neighbors with probability of broadcast $\mathrm{P}_{b}=1$. When an intermediate node first receives the RREQ packet, with probability $p_{b}<1$ it rebroadcasts the packet to its neighbors and with forwarding probability $1-$ $\mathrm{P}_{\mathrm{b}}$ it simply drop the packet. Since the decision of each node to rebroadcast a packet is independent, the possible number of rebroadcasts is $\mathrm{P}_{\mathrm{b}} \times\left(\mathrm{N}_{\mathrm{t}}-1\right)$.

Prediction of density of node in the network is not always feasible because of mobility speed. Under such conditions, fixed probabilistic route discovery [16] can suffer from a degree of inflexibility, since every node is assigned as fixed probability regardless of current status of the network. Forwarding probability should be high if a node located in a denser region compared with sparse region. Dynamic probabilistic route discovery approach is used to adjust the forwarding probability at a node based on local neighborhood information gathered [18]. If the number of neighbors is more than the average number of neighbors $\mathrm{Na}$ and such node is located at a dense region is considered. The neighborhood information is obtained by "hello" protocol to construct a 1-hop neighbor list at every node. A node that receives a hello packet from its neighbor node $\mathrm{N}$ periodically, creates an entry for $\mathrm{N}$ first time, else it updates the entry for $\mathrm{N}$. If there is no periodic hello for a particular node for with in time of threshold that node is no longer valid and removes the entry for $\mathrm{N}$ from its neighbor table. The hello interval [5] and its size can drastically consume the network resource and degrade the overall performance of the network. But the frequency of hello packets would be beneficial factor for the accuracy neighbor information. A size of 4 bytes and 2 bytes of hello packets with identification number respectively at a interval of 1.5 seconds is selected. Finally the probability of broadcast $P_{b}$ at a node is set low when relatively large percentage of its 1-hop neighbors are covered by the broadcast and region is considered as dense. Also, the probability $\mathrm{P}_{\mathrm{b}}$ is set high when small percentage of its neighbors is covered and region is considered as sparse and broadcast probability is adapts dynamically the at each node according to the number of neighbors.

\section{SIMULATION METHODOLOGY}

The simulation carried out with the Network Simulator (NS) version 2.34 event driven open source software on a platform with and Ubuntu 9.10. Network Simulator-2 (NS2 ) is extensively used in the research community. It is one of the most popular simulator developed by VINT project and it's a discrete event driven, object oriented network simulating tool, very much used by the researchers, professors and students. Simulation is the process of creating a model with its behavior. The table 1 shows the parameters fixed for entire simulation analysis of the four different approaches.

The four main performance metrics that substantially affect the performance of routing protocol are "throughput", "end to end delay", "Packet Delivery Ratio" (PDR) and "Routing Overhead" (RO). The throughput is measured by the total received size during the time elapsed between sending and receiving. A data packet experiences delay while crossing from source to destination including all possible delays caused by buffering during route discovery delay, queuing at the interface queues and retransmission delays at the MAC, propagation and transfer times known. This total is called as end-to-end delay. The packet delivery ratio is calculated from the ratio of number of data packets sent from the source number of data packets received at the destination.

TABLE.1. Simulation Parameters

\begin{tabular}{|l|l|}
\hline Parameter & Value \\
\hline Simulator & NS2(Version-2.35) \\
\hline Simulation area $\mathrm{x}(\mathrm{m})$ & $1500 \mathrm{~m}$ \\
\hline Simulation area y $(\mathrm{m})$ & $1500 \mathrm{~m}$ \\
\hline Transmission range & $250 \mathrm{~m}$ \\
\hline Mobility speed & $10 \mathrm{~m} / \mathrm{s}$ \\
\hline Number of nodes & $10,20,30,40,50$ \\
\hline Traffic type & CBR \\
\hline Mobility model & Random way point \\
\hline Packet rate & 8 packets/sec \\
\hline Packet size & 512 bytes \\
\hline Protocols & $\begin{array}{l}\text { AODV,DSDV,BNDS, } \\
\text { PBAODV }\end{array}$ \\
\hline Simulation time & $50 \mathrm{~s}$ \\
\hline
\end{tabular}


5 RESULTS AND DISCUSSION

5.1 Comparison of Throughput

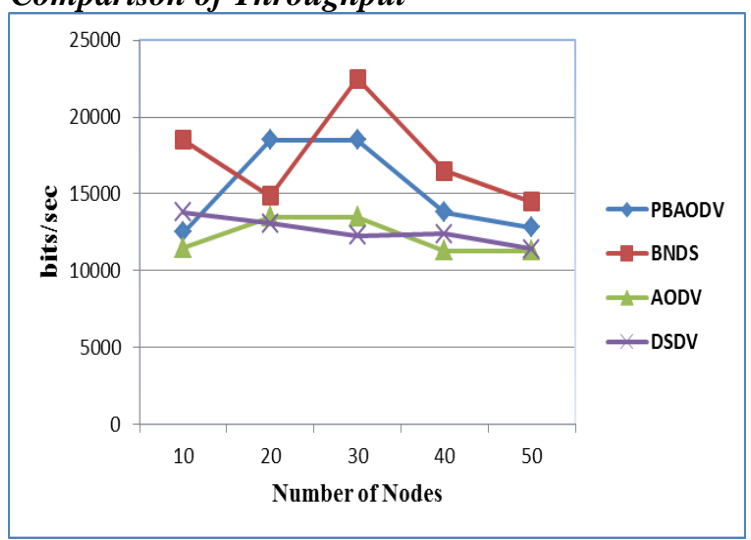

Fig. 1 Variation of throughput

Fig. 1 depicts the variation of throughput of AODV, DSDV, PBAODV and BNDS with variation in node density from 10 to 50 . It is evident that the BNDS and PBAODV approaches provide better average throughput than conventional routing protocol. The DSDV and AODV show closer performance with node density between 10 to 50 .

\subsection{Comparison of End to End Delay}

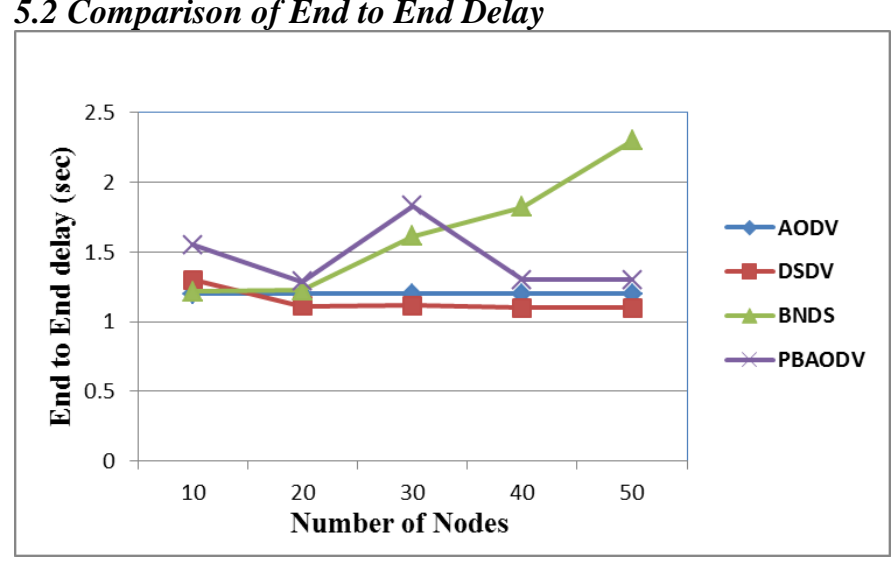

Fig. 2 Variation of end to end delay

Fig. 2 shows the variation of end to end delay of AODV, DSDV, PBAODV and BNDS for different node density. The results have revealed DSDV and AODV exhibit superior performance than proposed approaches. It is evident that among the three approaches DSDV consumes minimum delay.

\subsection{Comparison of Packet Delivery Ratio}

The bar chart shown in Fig. 3 shows the variation of packet delivery ratio for varying node density. It is seen that the delivery ratio for all the four routing protocols is greater than 65 percent. The BNDS and PBAODV have in general higher packet delivery ratio than other schemes.

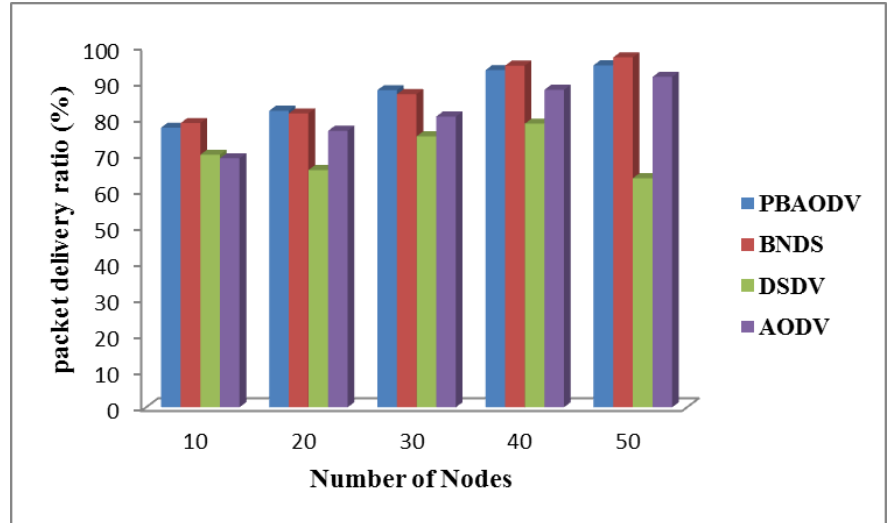

Fig. 3 Variation of PDR

\subsection{Comparison of Routing overhead}

Fig. 4 depicts the variation of routing overhead (in packets) of AODV, DSDV, PBAODV and BNDS for increasing node density from 10 to 50 and DSDV gives larger routing overhead other than the three approaches due to requirement of periodic routing updates . AODV has less routing overhead with node densities in the range of 10 to 20 nodes.

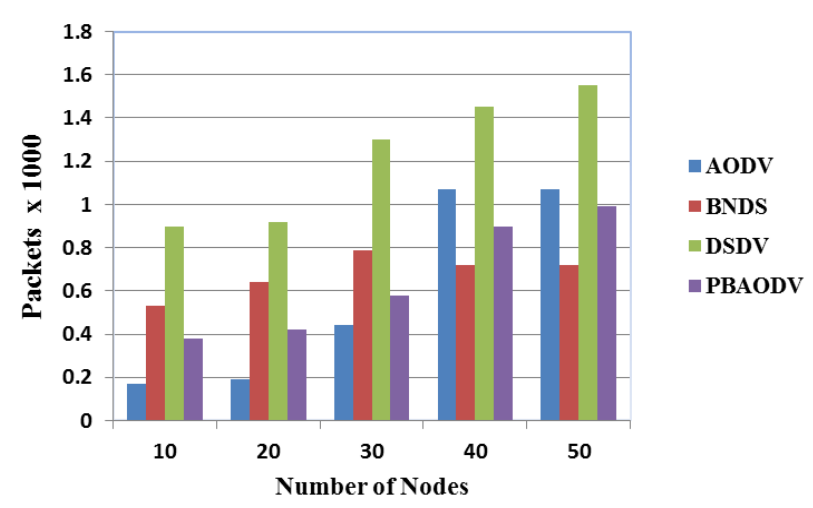

Fig. 4 Variation of RO

\section{CONCLUSION}

In this paper an effort has been made on the comprehensive examination and comparative study of four different new broadcasting approaches. The approach BNDS is a new broadcast neighbor discovery routing is implemented to reduce the overhead associated with flooding and to provide robust performance even with high traffic environments. In DSDV, the routing overhead increases with the frequency of routing updates. The PBAODV exhibits superior performance in terms of throughput, end to end delay and packet delivery ratio compared with other three approaches. DSDV has decreased PDR and increased routing overhead and nearly same throughput compared with AODV.

\section{REFERENCES}

[1] Abdalla, M., and Hanashi (2008), Improving Route Discovery of Dynamic Probabilistic Flooding in On-Demand Routing Protocols for MANETs, Proceedings of 10th Telecommunications Forum, pp. 89-92. 
[2] Abdulai, J., Ould-Khaoua, M., and Mackenzie, L.M., (2007), Improving Probabilistic Route Discovery in Mobile Ad Hoc Networks, 32nd IEEE Conference on Local Computer Networks (LCN 2007) IEEE Computer Society Press, pp. 739-746.

[3] Camp, T., Boleng,v., and Davies, V., (2002), A survey of mobility models for Ad hoc network research, Wireless Communications \& Mobile Computing(WCMC),Special issue on Mobile Ad Hoc Networking, Research,Trends and Applications, Vol. 2(5), pp. 483-502

[4] Williams, B., and Camp, T (2002), Comparison of broadcasting techniques for mobile ad hoc networks, Proceedings of the $3^{\wedge} \mathrm{rd}$ ACM International Symposium on Mobile Ad hoc Networking \& Computing, MOBIHOC, pp. 194 - 205.

[5] Cartigny, J., and Simplot, D (2003), Border node retransmission based probabilistic broadcast protocols in Ad-hoc networks, Telecommunication Systems, Vol.22(1), pp. 189- 204.

[6] Deepak. D., and Yogesh, C (2011), Traffic based analysis of efficient and dynamic probabilistic broadcasting algorithm in MANETs routing protocols, International Journal of Wireless and Mobile Networks, Vol. 3(01), pp. 108-116.

[7] Divecha, B., Abraham, A. , Grosan, C. ., and Sanyal, S., (2007), Analysis of Dynamic Source Routing and Destination- Sequenced Distance-Vector Protocols for Different Mobility models, Proceedings of First Asia International Conference on Modelling and Simulation, pp. 224-229.

[8] Perkins, C. E., and Royer, E.M (1999), Ad-hoc ondemand distance vector routing, Proceedings of the 1999 Second IEEE Workshop on Mobile Computing Systems and Applications, IEEE Computer Society, New York, pp. 90-100.

[9] Haas, Z., Halpern, J. Y., and Li, L., (2002), Gossipbased Ad hoc routing, Proceedings of IEEE INFOCOM '02, Vol. 21, pp. 1707-1716.

[10] Gnanamanoharan, E., and Bensraj, R. (2014), Performance evolution of proactive and reactive routing protocols in mobile Ad-hoc networks, International Journal of Research in Advent Technology, Vol. 3(03), pp. 92-97.

[11] Jagmeet Kaur., and Prabhjit Singh., (2016), Designing a Distributed Framework to Detect a Selfish Node in MANET by using a Collaborative Approach, International Journal of Computer Science Trends and Technology, Vol. 4(04).

[12] Jose Moses, G., Sunil Kumar, D., Suresh, P., Varma., and Supriya, N., (2012), A Simulation Based Study of AODV, DSR, DSDV Routing Protocols in MANET Using NS-2, International Journal of Advanced Research in Computer Science and Software Engineering, Vol.02 (03), pp 43-51.

[13] Kim, J.S., Zhang, Q., and Agrawal, D. P., (2004), Probabilistic broadcasting based on coverage area and neighbour confirmation in mobile ad hoc networks, Proceedings of IEEE Global

Telecommunications Conference Workshops (GlobeCom'2004), pp . 96-101.
[14]Manickam, P., Guru Baskar, T., Girija, M., and Manimegalai, D., (2011), Performance Comparisons Of Routing Protocols In Mobile Ad Hoc Networks, International Journal of Wireless \& Mobile Networks (IJWMN), Vol. 03 (01), pp. 98-106.

[15] Mehmood, Z, (2014), Comprehensive experimental performance analysis of DSR, AODV and DSDV routing protocol for different metrics values with predefined constraints, International Journal of Information technology and computer science, pp. 24-31.

[16] Mohammadizadeh., Moyaghar, A., and Safi, M., (2009), SEAODV: Secure Efficient AODV Routing Protocol for MANETs Networks, Proceedings of 2nd International Conference on Interaction Sciences: Information Technology, Culture and Human, Seoul, Korea, Vol.24, pp. 940-944.

[17] Gnanamanoharan, E., and Bensraj, R. (2016), Performance analysis of broadcast neighbors discovery protocol for mobile Ad-hoc networks based on node mobility, International Journal of Innovation and Scientific Research, Vol. 25(01), pp. 352-359

[18] Gnanamanoharan, E., and Bensraj, R. (2016), Performance analysis of probabilistic broadcast on demand route discovery protocol for mobile Ad-hoc networks based on node mobility, International Journal of Advanced Engineering Technology, Vol. 07(03), pp. 174-178.

[19]Ni, S.Y., Tseng, Y.C., Chen, Y.S., and Sheu, J.P. (1999), The broadcast storm problem in a mobile Ad hoc networks, Proceedings of the $5^{\wedge}$ th Annual ACM/IEEE International Conference on Mobile Computing and Networking, pp.152-162.

[20] Gnanamanoharan, E., and Bensraj, R. (2014), Impact of variable bit rate and packet size on the performance evaluation of neighbor aware AODV and DSDV routing protocols for MANETs, International Journal of Computer Applications, Vol. 92(08), pp. 43-47.

[21] Perkins, C. E., and Bhagwat, P (1994), Highly dynamic destination-sequenced distance vector routing (DSDV) for mobile computers, Proceedings of ACM SIGCOMM 94, pp. 234- 244. 